\title{
Over-The-Air (OTA) Testing 5G Wirelesses Communications Systems
}

\author{
Mounia Belhabib
}

\begin{abstract}
The need for high data-rate, reliable and low latency transmission in wireless communication systems motivated a multitude of wireless standards, spanning from 3G-4G, WLAN to the upcoming fifth generation (5G) of mobile networks. In this context, technology providers, as well as operators, need to develop accurate and cost effective standard test methods, to evaluate devices performance. Over-The-Air (OTA) test methodologies aim to reproduce radio multipath environments in laboratory in repeatable and controllable manner, avoiding costly field test. The focus of this paper is on multi-probe anechoic chamber approach. Section II is devoted to describe the setup of the testing methodologies. Their advantages and disadvantages are discussed. Multi-probe Over-the-air is the methodology selected for $5 \mathrm{G}$ systems.
\end{abstract}

Index Terms-Over-The-Air, 5G, MIMO system, emulator fading.

\section{INTRODUCTION}

In the last decades, wireless telecommunications did not cease to evolve. On the one hand a wide variety of advanced technologies have greatly facilitated our daily lives, on the other hands the user needs stimulated an explosive growth of these technologies.

In the years 2000 the third generation (3G) of mobile phone network, which is based mainly based on Universal Mobile Telecommunications System (UMTS) and CDMA2000 technology, has been deployed to provide up to $42 \mathrm{Mbit} / \mathrm{s}$ data rate. However, the demands of users on the quality and efficiency of networks make challenges to invent new systems.

As a result, the fourth generation (4G) mobile radio system is developed. The prime objective of $4 \mathrm{G}$ mobile system is to achieve a fully integrated digital communication that offers voice, data, and multimedia, and to provide enhanced peak data rate of $100 \mathrm{Mbit} / \mathrm{s}$. The Long Term Evolution (LTE) standard was first proposed by 3GPPP to fulfill the $4 \mathrm{G}$ requirements. Finally, only in 2010, the International Telecommunication Union (ITU) recognized the LTE Advanced (LTE-A) as 4G technology. The 3GPP rel.11 foresees bandwidth from $1.4 \mathrm{MHz}$ to $20 \mathrm{MHz}$, and 64 QAM modulation providing up to $450 \mathrm{Mbit} / \mathrm{s}$ downlink data rate. One of the key-technologies employed is Multiple Input Multiple Output (MIMO) approaches, exploiting multi-antenna system. Nowadays the focus of the research and development is on the fifth generation $(5 \mathrm{G})$ of wireless communication, which is expected to answer to a wide

Manuscript received November 6, 2019; revised February 12, 2020.

Mounia Belhabib is with Intel Corporation, France (e-mail: belmounia40@gmail.com). number of use cases requirement, spanning from the Internet of Things (IoT) to high-data rate and low-latency communication. It promises significant gains in wireless network capacity and data rates up to $20 \mathrm{Gbits} / \mathrm{s}$. Different technologies are aimed for the development of 5G, including massive MIMO and millimeter wave. However the first technologies deployed are expected to work for frequency below $6 \mathrm{GHz}$. Also vehicle-to-everything (V2X) communications are expected to gain an important role in the next years. Here the data volume is relatively low, and the foreseen standard is an evolution of Wireless Local Area Network (WLAN) based on IEE 802.11(p) working at frequencies around $5.9 \mathrm{GHz}$ [1]. At the beginning, in section 2 , the principle of OTA testing is described. In section 3, the next wireless generation system $(5 \mathrm{G})$ are identified. One of the challenges in the research and development of $5 \mathrm{G}$ antenna systems is the performance evaluation methods. SectionII presents a critical review of different OTA methodologies; reverberation chamber, two stage methods and anechoic chamber method. The questions raised in analyzing these methodologies will be discussed and answered in section III the selected studies are discussed in sectionV. Although multi-probe method is comparatively costly, from technical points of view it appears to be the most flexible one for $5 \mathrm{G}$ wireless performance evaluation.

\section{OVER-THE-AIR (OTA)}

OTA testing solutions create an environment where signals are transmitted to verify the performance of a product and accurately distinguish a poorly performing device from a good device. OTA test methods evaluate the wireless device end to end. If the device has a single antenna mode, it can be evaluated using traditional SISO OTA measurements methods. In all other cases, new test methodologies metrics are required to correctly characterize the device under test.

\section{A. Principle}

The first SISO OTA specification developed by The Cellular Telecommunications Industry Association (CTIA) released in 2001 and later by $3 \mathrm{GPP}$ [2]. It defines the general requirement for equipment configurations, laboratory techniques, test methodologies, and evaluation criteria that must be met in order to ensure the accurate, repeatable, and uniform testing of wireless devices. Proposed metrics for device radiation performance evaluation are Total Radiated Power (TRP) and Total Isotropic Sensitivity (TIS). TRP metric is a metric that provides information about radiated RF performance of the DUT. 


\section{B. Single Input Single Output OTA Tests}

The TRP metric is calculated by computing the average of the radiated power over a sphere centered on the device under test (DUT). The TIS metric is the average over the same sphere of the minimum received power to achieve a particular bit error rate. This DUT receiver measurement is made while the DUT is transmitting at maximum power so that any radiated effects that might cause self-blocking or desensitization of the DUT receiver are fully captured. Most of the work in this field was then concentrated on defining the right measurement procedure, the uncertainty of the technique and the minimum. DUT performance demanded. The maximum uncertainty allowed is defined in and showed in.

For the operators and manufacturers of mobile industry there is a need to evaluate, by standardized measurements, the performance of such advanced systems This evaluation must be carried out in realistic way in different representative environment such as indoor or outdoor. However, the field tests in the application environments are tedious and costly. For this reason, the so called Over-The-Air (OTA) channel emulation technique appears as a reasonable solution, for reproduce repeatability and reliability realistic fading conditions.

OTA test methodologies aim to recreate in a laboratory the multipath environment, to characterize and evaluated wireless devices. It can include the actual interaction of the antennas, radio frequency front ends and baseband processing elements. OTA is the best way to evaluate the performance as experienced by the user equipment. OTA testing terminal has attracted great attention by the research community in the last years. However a great number of questions are still open to reproduce accurately the radio channel.

\section{5G WIRELESS COMMUNICATION}

The next generation wireless radio technologies, such as fifth generation $5 \mathrm{G}$ is under intensive development. The focus on delivering higher level of throughput. The applications of $5 \mathrm{G}$ communication include cloud computing, Mobile Internet of Things (IoT) for 5G communications [2].

In $5 \mathrm{G}$, there exists 1000 times increase with respect to the capacity compared to the current wireless communication networks, which consists of 10 times growth in data rate, 10 times growth in spectral efficiency and 10 times growth in energy efficiency. With that, the industry is moving from the early $5 \mathrm{G}$ technology commercialization and deployment phase. One key issue for this technology is possibility to integrate a large number of antennas. Massive MIMO is systems are considered essential in contributing to the increasing a spectral efficiency. RF testing signals can be therefore carried to the antennas systems directly with the RF longer applicable for $5 \mathrm{G}$ devices systems..

\section{OTA Test Methodologies}

OTA becomes very important for testing MIMO systems or products in real conditions. Ranging devices as good fair or poor w.r.t is a common practice in the industry. One distinguishes three categories of method for such MIMO OTA characterization:

1) Reverberation chamber based method.

2) Two-stage method.

3) Anechoic chamber based multi-probes OTA method

\section{A. Reverberation Chamber}

Because the final formatting of your paper is limited in scThe Reverberation Chamber is a metallic cavity or cavities that can emulate an isotropic multi-path environment which represents a reference environment for systems designed to work during fading; similar to how the free space "anechoic" reference environment is used for tests of Line-Of-Sight systems. The Rayleigh environment in a reverberation chamber is well known as a good reference for urban and indoor environments, but does not well represent rural and suburban environments. A typical mode stirred reverberation chamber (MSRC), also referred as Reverberation Chamber (RC), consists of a closed metal cavity equipped with movable metal blades that act as field mixers (commonly called stirrers) and a turntable that rotates the DUT. A description is provided in Fig. 1 [3]. The independent movement of the stirrers and the rotation of the sample, allow to dynamically changing the boundary conditions of the electromagnetic field generated inside the cavity. In a perfectly agitated MSRC, the real and imaginary parts of the rectangular components of electric and magnetic fields follow Gaussian distributions with identical paths variance. Thus, the amplitude of the electric and magnetic fields measured in a perfectly stirred follows a Rayleigh probability density function (PDF), while the phase is uniformly distributed. This type of distribution is very similar to the multipath field distribution found in urban environments for mobile communication systems. The naturally emulated Rayleigh environment in a MSRC is well known as a good reference for urban and indoor multipath environments, but does not reflect the reality of other propagation environments. Limits to emulate arbitrary channel models in a typical MSRC can be summarize as:

1) Delay Spread (DS) cannot be arbitrarily set.

2) Doppler is limited by the relatively slow motion of the stirrers.

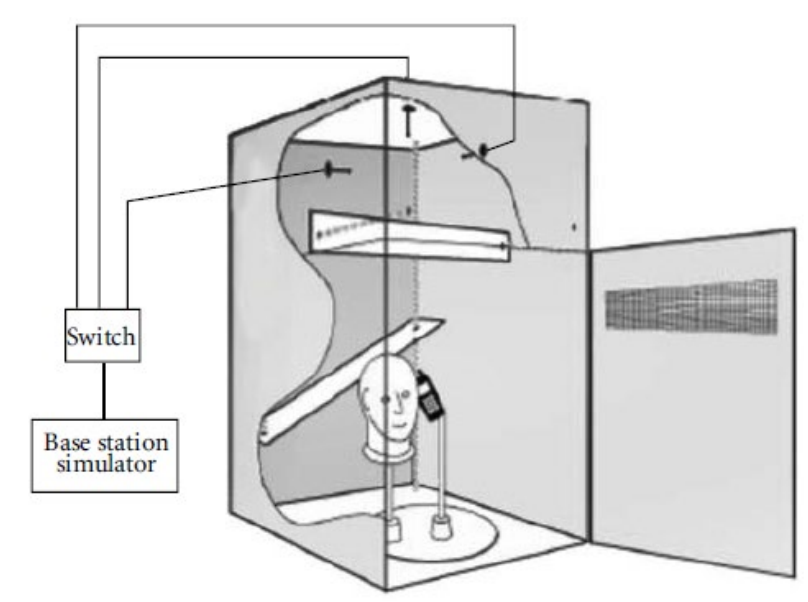

Fig. 1. Reverberation chamber setup for devices testing with single cavity. 
On the other hand, there are some important advantages that have made this methodology become an emerging technique during the last few years.

1) Cost effective solution: the number of probe antennas used in a MSRC is much lower than in other solutions (as those based in anechoic chamber).

2) Measurement time: due to the capability of MSRC to emulate a 3-D environment (instead of 2-D environments generated with other methodologies), the measurement time with this technique is lower than in other techniques and can be even suitable to be included in production lines.

\section{B. Two Stage Method}

The two stages method consists on the measurements of far field antenna radiation patter therefore the method divides the MIMO OTA tests in two separate measurements. Firstly, the MIMO antenna radiation pattern is measured inside an anechoic chamber. Secondly, a commercial channel emulator is used to mathematically convolve the measured antenna characteristic with the chosen OTA channel model for real time emulation. In [4] authors explains how to experiment this methodology in the anechoic chamber. They demonstrated that the first stage consists of the characterization of the three dimensional antenna radiation patterns of all the antennas in the DUT, using an anechoic chamber. The setup needed to perform this part of the measurement is defined in the 3GPP document TS 34.114. The measurements are started by placing the DUT in the anechoic chamber in the far zone radiation pattern. The measurements were conducted in two polarizations as shown in Figure [2], [3]. The user influence can be included in this stage, by repeating the antenna radiation pattern characterization including head and hand phantoms.

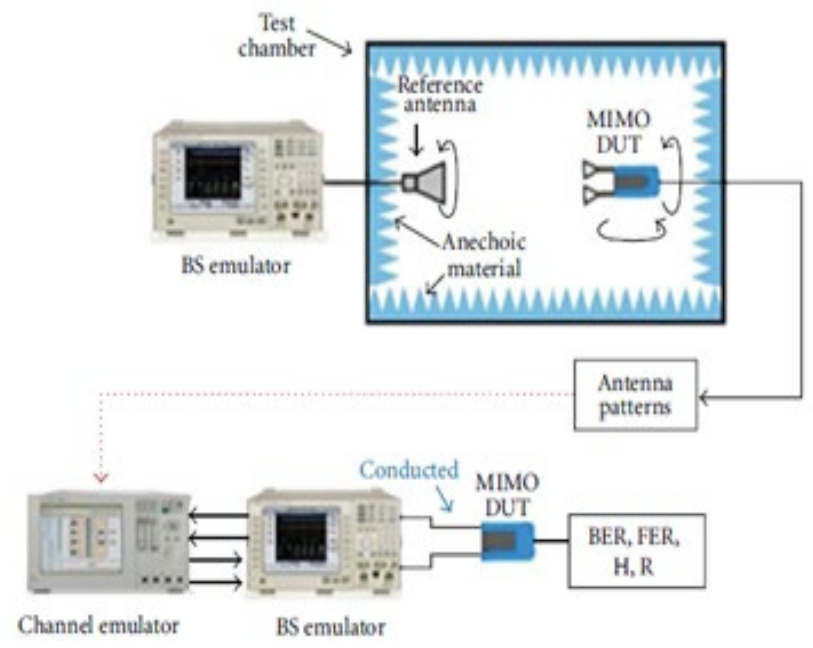

Fig. 2. Proposed two-stage test methodology for MIMO OTA test.

The second stage of this method consists on performing the desired FoM conducted measurements. To establish the communication, the BSE is connected to the MIMO CE (emulating the compound channel, including both the MIMO antenna effect and the multipath channel effect) and then to the MIMO DUT's temporary antenna ports via approved RF cables, as it is showed in Fig. 3. By controlling the power settings of the channel emulator and also the integrated channel model, the end-to end throughput with the MIMO antenna radiation influence can be measured [5].

\section{1) Multi-probe and fading emulator}

His type of OTA test methodology groups a number of slightly different methodologies, but all of them include an anechoic chamber and several antennas that surround the DUT. These techniques are commonly referred as anechoic chamber based methodologies [5]. These methodologies consist of a large number of probe antennas placed inside an anechoic chamber transmitting with temporal and spatial characteristics for testing multiple antenna devices. Probe antennas are positioned around the DUT in such a way that it is possible to change the receiving spatial profile of Angle of Arrival (AoA) and consequently the Angle Spread (AS) at the DUT position. A schematic representation of the setup of a typical multi-probe method for MIMO OTA testing facility is illustrated in Fig. 3.

The spatial fading emulator presented by Tokyo Institute of Technology and Panasonic [3] has been further developed in parallel at SP and Sony Ericson [6]. Various aspects and measurements have been presented in COST 2100 Transmit Diversity (TD) s (see 3). It is shown for the SFE has 62 dipoles antennas in 31 pairs dual polarized. Phase shifters, power dividers and attenuators, operating in the RF band, it has been shown that a realistic fading channel environment can be emulated reduce the influence from the measurement equipment [7].



Fig. 3. Spatial fading emulator test setup.

\section{Multi-Probe OTA StSEM}

Multi-probe setup in anechoic chamber, combined with fading emulator, are nowadays considered as an attractive solution for Over-The-Air (OTA) test of mobile communication devices. The multi-probes test methodology employs a number of spatially separated antennas placed in anechoic chamber and combined with a fading emulator, in order to reproduce the channel characteristics in a controlled and repeatable manner. The probes are fed by signals, which are jointly optimized in order to generate a superposed field impinging on the Device under Test (DUT), as in the real radio environment. Most of contributions in literature focuses on two-dimensional setup by considering a single 
OTA ring of antennas. Recently three-dimensional (3D) OTA setups have been investigated [8], [9], proposing different probe configurations and analyzing the effect on channel emulation accuracy. In [10] it was shown that 32 symmetrically placed OTA antennas in a 3D setup are able to reproduce $3 \mathrm{D}$ channels models, while a configuration of a spherical layout is discussed in [10]. Authors presented the result, based on Pre-Faded Signal Synthesis (PFS), with limited number of probes in a theoretical 3D configuration. In [10] authors built an experimental setups based on the multipoles anechoic chamber, the test setup was two dimensional ring of probes around (DUT) in OTA ring $\mathrm{R}=$ $2 \mathrm{~m}$. The measurements uncertainty investigation with a fading emulator in the proposed test setup was presented in [11]. In [12] we presented a comparison simulation of cylindrical and spherical (3D) MIMO OTA multi-probes configurations. Spatial correlation is used as a metric to evaluate the setup accuracy, but not experimental realization was given. In this work we present a novel 3D hemispheric OTA test-bed able, that can be used in a wide span of applications from 2 to $6 \mathrm{GHz}$. The characteristics of the OTA test-bed depend on the frequency as well as the OTA radius of the chamber.

\section{CONCLUSION}

In this paper we provided an overview of the different approaches and techniques to test devices performances exposed to different radio propagation channel models. The first approach which has been presented is the two stage method which cannot really be qualified as fully "over the air" and two other approaches "fully over the air" which exploits different kind of confined environments either reverberant or anechoic.

The two-stage method has the problem with the cable connection to the antenna ports it requires, which means that the test is never performed in the realistic usage conditions of the device under test. The difficulty with reverberation chamber-based methods is that they are not well suited for synthesizing all channel conditions in line with usual geometric radio channel model. From these technical points of view the anechoic chamber based methods appear to be the most promising method for device performance evaluation. For this reasons we adopted the multi-probe anechoic chamber based method to test $5 \mathrm{G}$ wireless systems. This choice is made for several reasons: the ability to emulate a variety of fading scenarios, especially geometrical ones and the cost effectiveness of the work or suggest applications and extensions.

\section{CONFLICTS OF INTEREST}

The authors declare no conflict of interest.

\section{AUTHOR CONTRIBUTIONS}

The principal author contributes in this paper. Théoretical studies are invistigated. Several comparison are established in order to present a good solution for $5 \mathrm{G}$ test methodologies.

\section{REFERENCES}

[1] A. Osseiran, J. F. Monserrat, P. Marsch, M. Dohler, and T. Nakamura, $5 G$ Mobile and Wireless Communications Technology, Cambridge University Press, July 2016.

[2] E. G. Larsson, O. Edfors, F. Tufvesson, and T. L. Marzetta, "Massive mimo for next generation wireless systems," IEEE Communications Magazine, vol. 52, no. 2, pp. 186-195, February 2014.

[3] 3GPP, Technical Specification Group Radio Access Network Measurements of Radio Performances for UMTS Terminals in Speech Mode (Release 11), Jun. 2015.

[4] 3GPP, Verification of Radiated Multi-antenna Reception Performance of UEs in LTE/UMTS, RP-120368, 2012.

[5] 3GPP, Technical Specification Group Radio Access Network Measurements of radio Performances for UMTS Terminals in Speech Mode (Release 7), Jun. 2011.

[6] T. Sakata, et al., "Experimental investigation of a dual-band handset MIMO antenna using a spatial fading emulator," in Proc. Antennas and Propagation Society International Symposium (APSURSI), 2010, pp. 1-4.

[7] Air Interface Working Group; Verizon 5th Generation Radio Access; Physical Layer Procedures, Verizon 5G TF, Tech. Rep. TS V5G.213 v1.0 (2016-06), June 2016.

[8] P. Kyosti and A. Khatun, "Probe configurations for 3D MIMO Over-theAir testing," in Proc. 7th European Conference on Antennas and Propagation (EuCAP), 2013, pp. 1421-1425.

[9] W. Fan, P. Kyosti, S. Fan, J. Nielsen, X. Carreno, G. Pedersen, and M. Knudsen, "3D channel model emulation in a MIMO OTA setup," in Proc. IEEE 78th Vehicular Technology Conference (VTC Fall), 2013.

[10] W. Fan, I. Carton, P. Kysti, and G. F. Pedersen, "Emulating ray-tracing channels in multiprobe anechoic chamber setups for virtual drive testing," IEEE Transactions on Antennas and Propagation, vol. 64, no. 2, pp. 730-739, Feb 2016.

[11] W. Fan, I. Szini, M. D. Foegelle, J. Nielsen, and G. F. Pedersen, "Measurement uncertainty investigation in the multi-probe OTA setups," in Proc. the 8th European Conference on Antennas and Propagation (EuCAP 2014), 2014, pp. 1068-1072.

[12] M. Belhabib, R. D. Errico, and B. Uguen, "Spatial correlation in spherical and cylindrical 3D MIMO over-the-air tests setups," in Proc. 10th European Conference on Antennas and Propagation (EuCAP), April 2016, pp. 1-4.

Copyright (C) 2020 by the authors. This is an open access article distributed under the Creative Commons Attribution License which permits unrestricted use, distribution, and reproduction in any medium, provided the original work is properly cited (CC BY 4.0).

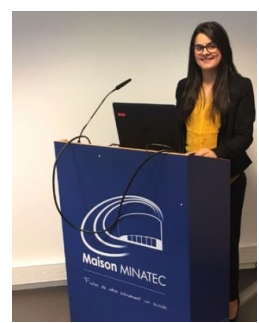

Mounia Belhabib received her Ph.D. degree in Radio Frequency and Telecommunications systems from CEA LETI Grenoble and IETR Rennes France in 2017. She is currently Research R\&D Engineer at Intel Corporation, France. She is head of Wireless communication systems department. She is working in collaboration with International companies (Samsung, Apple) in developing and certifying a next $5 \mathrm{G}$ Generation of wireless devices. Her main areas of my research is MIMO Over-The-Air Testing for $5 \mathrm{G}$ wireless communications systems. In this context, technology providers, as well as operators, need to develop accurate and cost effective standard test methods, to evaluate devices performance. Over-The-Air (OTA) test methodologies aim to reproduce radio multipath environments in laboratory in repeatable and controllable manner, avoiding costly field test. The focus of this thesis is to propose a new OTA test methodology, in order to emulate radio channel propagation, over a wide band, and to evaluate the performance of the wireless systems in real environments. She contributed with IEEE publications in this context and also participate at different Industrial workshops. 